\title{
Dread and latency impacts on a VSL for cancer risk reductions
}

\author{
Rebecca L. McDonald ${ }^{1}$ • Susan M. Chilton ${ }^{2}$. \\ Michael W. Jones-Lee ${ }^{2}$ Hugh R. T. Metcalf ${ }^{2}$
}

Published online: 26 April 2016

(C) The Author(s) 2016. This article is published with open access at Springerlink.com

\begin{abstract}
We propose a structural relationship between the value of preventing a statistical cancer fatality and the value of statistical life (VSL) for risks of an instantaneous road accident fatality. This relationship incorporates a context effect reflecting both the illness or 'morbidity' associated with cancer fatality and the 'dread' or horror associated with the prospect of eventual death from cancer, as well as a latency effect that captures the discounting likely to arise because the onset of the symptoms of cancer typically occurs after some delay. We use a Risk-Risk trade-off study to validate this model by directly estimating the influence of context and latency effects upon the relative size of the VSL for cancer and for road accidents, confirming that both effects are significant and estimating their size using regression analysis. We show that morbidity accounts for the majority of the context premium. We use the elicited coefficients to reconstruct VSL estimates for a range of cancers characterised by their latency and morbidity periods.
\end{abstract}

Keywords VSL $\cdot$ Cancer-Risk-Risk trade-off

JEL Classification J17

This article and the work it describes were funded by the Health and Safety Executive (HSE). The article's contents, including any opinions and/or conclusions expressed, are those of the authors alone and do not necessarily reflect HSE policy. The paper was also supported by the Economic and Social Research Council, UK [grant number ES/K002201/1] and the Leverhulme Trust [grant number RP2012-V-022].

Hugh R. T. Metcalf

hugh.metcalf@newcastle.ac.uk

1 University of Warwick, WBS Scarman Road, Coventry CV4 7AL, UK

2 Newcastle University Business School, Newcastle University, 5 Barrack Rd., Newcastle NE1 4SE, UK 


\section{Introduction}

The Value of Statistical Life ${ }^{1}$ (VSL) reflects the monetary value to the affected population of a small reduction in the risk of premature death (Jones-Lee 1989). In the UK, it is conventionally defined as the aggregate willingness to pay by a large, representative sample of individuals for small reductions in the risk of death which, taken across the group concerned, will reduce the expected number of fatalities during a forthcoming period by one and will hence save one "statistical life". As such, the VSL will clearly be determined by the marginal rate of substitution (MRS) of wealth for risk of death for individuals within the affected group, and for a group enjoying equal marginal risk reductions, it will be equal to the arithmetic mean MRS for the group concerned - see, for example, Jones-Lee (1989) and Viscusi (1998). By contrast, in the USA, the term is typically applied directly to the MRS. Both definitions clearly share the same conceptual foundations. In the study reported in this paper, we analyse the data at the individual level which is akin to the US approach, although for UK policy extrapolation we aggregate the data to conform with the UK definition and application of the VSL.

In the UK, the (aggregated) VSL was initially implemented in the transport sector (Jones-Lee et al. 1985) and elicited in this context. However, increasingly there has been a policy-driven trend to extend its use to other sectors, both in the UK and elsewhere, as has been noted by the Organisation for Economic Co-operation and Development (OECD 2012). Whilst intuitively we might expect contextual factors including the mode of death to affect the perceived value of risk reductions, governments generally apply the same VSL across contexts. The exception is when the change in risk applies to cancer fatalities, as exemplified by the UK Health and Safety Executive (HSE)'s 2001 current recommendation for a cancer VSL. ${ }^{2}$

'Where the benefit is the prevention of death, the current convention used by HSE, when conducting a CBA is to adopt a benchmark value of about $£ 1,000,000$ (2001 prices) for the value of preventing a fatality (VPF). This is the VPF adopted by the Department of Transport, Local Government and the Regions for the appraisal of road safety measures. It may well be the case that individuals' willingness to pay for risk reduction - measured in aggregate by the VPF — will vary, depending on the particular hazardous situation. Thus, the particular hazard context will need to be borne in mind when a VPF figure is adopted. Currently, HSE takes the view that it is only in the case where death is caused by cancer that people are prepared to pay a premium for the benefit of preventing a fatality and has accordingly adopted a VPF twice that of the roads benchmark figure. Research is planned to assess the validity of this approach.' (HSE 2001)

Applying a cancer premium to the VSL is principally intended to reflect the fact that death from cancer is typically preceded by a protracted period of ill-health and dread caused by the prospect of imminent fatality. In addition, evidence from the first stated

\footnotetext{
${ }^{1}$ Also termed the Value of Preventing a Statistical Fatality (VPF) in the UK.

${ }^{2}$ The Directorate-Generale of the EU takes a similar position, recommending a cancer premium of 1.5 (see European Commission 2001).
} 
preference-based VSL study conducted in the UK indicated that as far as members of the public were concerned, willingness to pay (WTP) for a given reduction in the number of deaths from cancer was almost double the corresponding amount for reducing the number of road accident deaths by the same figure - see Jones-Lee et al. (1985). This position is supported by economic theoretic and psychological evidence which suggests that willingness to pay for risk reductions may naturally be expected to vary across causes, for example because of differing baseline exposure risks (Pratt and Zeckhauser 1996; Covey 2001) or personal risk perception (Slovic 1987; McDaniels et al. 1992). When well-informed and carefully considered survey responses generate a VSL that incorporates a premium for certain types of fatality, then a strong case can be made that the VSL figure used in policy making should reflect this premium.

\section{Context, latency and cancer risks}

Nevertheless, two important reservations can be made with respect to the upward adjustment of the VSL for cancer (hereafter $\mathrm{VSL}_{\mathrm{CANCER}}$ ). The first relates to the fact that death from cancer will not take place immediately even if exposure to this risk is in the current period. This is due to the latency period in the development of most cancers. Latency is defined as the delay between an individual's exposure to the conditions that are the basic cause of cancer and the onset of symptoms, with death occurring after a period of illness referred to as the "morbidity period". Recognising latency is important in the policy process, because typically a policy is implemented in the current period to reduce the risk of developing and dying from cancer in the future. For example workplace policies often reduce the exposure of workers to radiation or other carcinogens, and air pollution reduction measures implemented now reduce the risk of developing lung cancer in the future. Economic theory (and empirical evidence) suggests that people discount future events relative to present events (Samuelson 1937; Frederick et al. 2002), in part due to the inherent uncertainty about reaching that future point, but also due to an intrinsic preference for good things to happen now, and bad things later. Epidemiological evidence indicates that this latency delay will typically be in the region of 5-25 years. ${ }^{3}$ When comparing the VSL for cancer with the figure applicable to road fatalities it therefore seems likely that to some extent latency delays will mitigate the context effects referred to earlier. Valuations from studies that do not incorporate a latency period into the cancer description will be unable to capture this (potentially) offsetting effect and so using these valuations to inform policies that affect latent cancer risks could result in overvaluing the avoidance of future cancer fatality risks relative to the avoidance of fatality risks in the current period. The extent to which various empirical studies control for latency has been explored in Viscusi (2013) and the need for more comprehensive treatment of future risks has recently been considered by Cameron and DeShazo (2013).

The second reservation stems from the lack of conclusive evidence in the literature either for or against the application of a cancer premium. ${ }^{4}$ A number of studies use information on revealed preferences, typically using data on house prices and environmental cancer risks - for example, Gayer et al. (2000); Gayer et al. (2002), and Davis

\footnotetext{
${ }^{3}$ These figures refer to occupational cancers for which official data are available (Rushton et al. 2010).

${ }^{4}$ This review is by necessity brief. For a more comprehensive discussion see Viscusi (2013).
} 
(2004). On the basis of these three studies, there is no evidence to suggest that latent cancers are valued at a premium compared to instant road accident fatalities.

Turning to the stated preference literature, evidence in support of a cancer premium can be found in an early study by Van Houtven et al. (2008) as well as in Alberini and Scasny (2010) and (2011). These studies find, respectively, premia of 3:1 (for a latency of 5 years, dropping to 1.5 when the latency period increases to 25 years in the US), 2:1 (Italy) and 2.5:1 (Czech Republic). Viscusi et al. (2014), holding latency constant at 10 years, find evidence of a statistically significant cancer premium of $21 \%$.

In contrast, a number of studies find no evidence of a significant cancer premium. An early example by Magat et al. (1996) finds a cancer to roads VSL relativity of 1:1. They include detailed morbidity descriptions but do not specify any latency. Hammitt and Liu (2004) directly test the effects of disease type and latency and find that while latency (20 years) has a significant effect on willingness to pay for risk reductions, there is no statistically significant effect of cancer. ${ }^{5}$ Hammitt and Haninger (2010) find no significant difference between the values of risk reductions for cancer compared to any other cause, including road accidents, in the US. Adamowicz et al. (2011) find that cancer risk reductions are in fact valued less than microbial disease risk reductions.

Finally, it should not be surprising, given the above discussion, that between the group of studies that support a cancer premium and the group that finds evidence against one, there is a range of studies that find mixed or inconclusive results on the matter (Tsuge et al. 2005, Japan; Cameron et al. 2009, US).

A summary of the stated preference studies, including information about the latency and morbidity periods involved, is provided in Table 1 . Notice that while some studies reported terminal and curable cancers, where we provide a value for the premium it is for fatal cancer compared to instant road accident fatality. Clearly, the studies differ in methodology and country, but also in the information respondents were given, particularly regarding morbidity and latency.

Some of the studies examined do not specify for respondents what kind of cancer is to be considered, which means that respondents are left to imagine the symptoms, which then lie outside of the researchers' control. Examples are Alberini and Scasny (2010) and Alberini and Scasny (2011) who give "basic information" and Tsuge et al. (2005). A full meta-analysis of stated preference studies (OECD 2012) did not find evidence to suggest a cancer premium, instead recognising that the morbidity preceding fatality generates a higher value for cancer risk reductions, but recommending that morbidity costs prior to death should be added separately as opposed to being incorporated in a cancer premium.

More recent research by Cameron and DeShazo (2013) recognises the heterogeneity of fatality risk scenarios and explicitly addresses latency and morbidity in a choice experiment where the scenarios vary along these dimensions. Their results suggest that both latency and morbidity are important in explaining the VSL, with longer morbidity prior to fatality engendering higher WTP to avoid the risk than shorter morbidity, and that for older members of the sample, VSL for latent fatality is lower than for more immediate fatality risks. They do not, however, report the case of cancer explicitly, instead using cancer scenarios for describing the profiles but reporting aggregated results across illnesses.

\footnotetext{
${ }_{5}^{5}$ Hammitt and Liu (2004) find a premium of around one-third, but it is not statistically significant at conventional levels.
} 


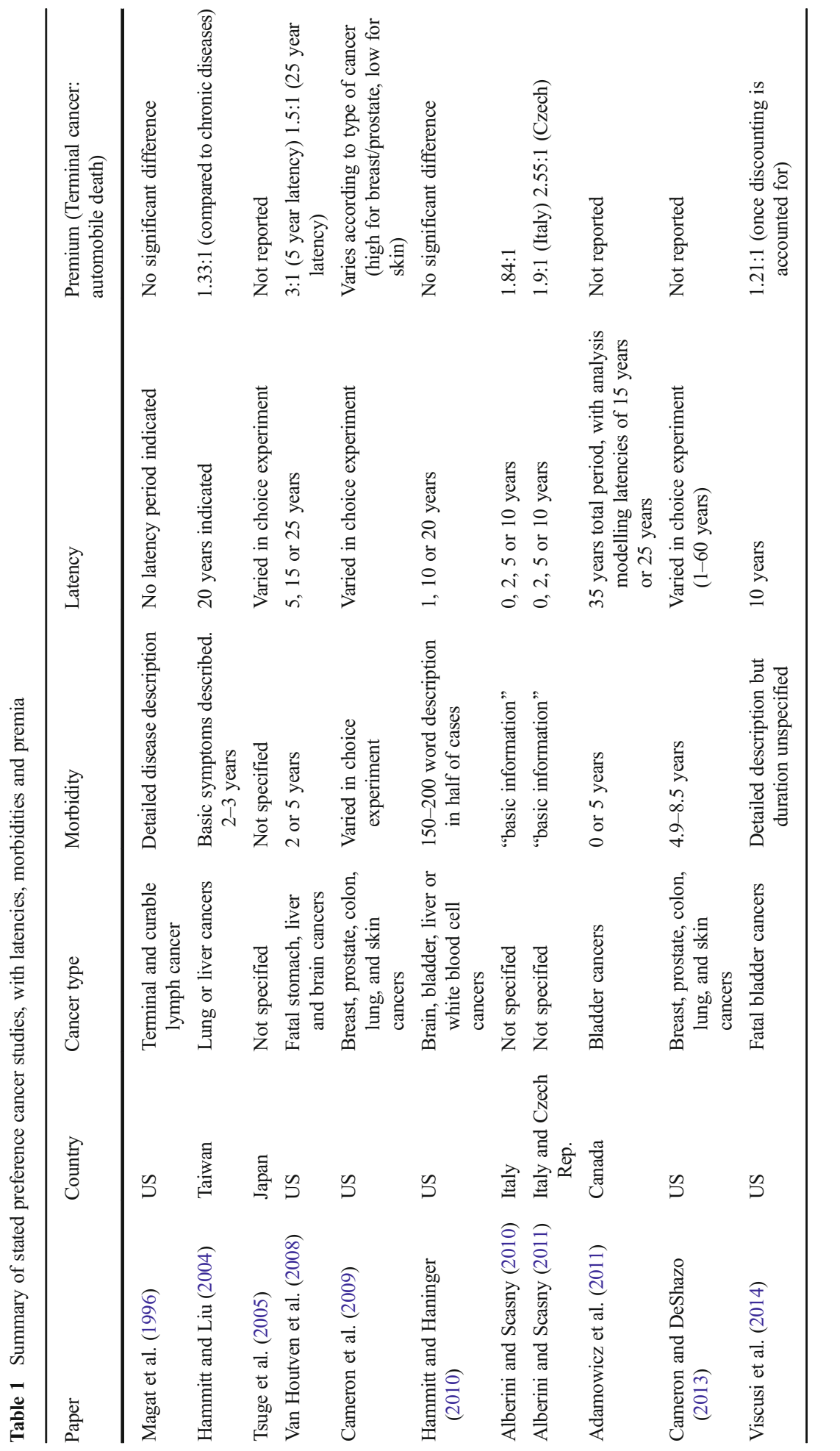


Based on the review of the literature, we propose that the lack of consensus in the cancer VSL literature may reflect the different information that is presented to respondents about the latency periods associated with cancer. While techniques are well developed for eliciting the overall relativity for a latent cancer fatality compared to a road accident fatality, with just a handful of exceptions, limited progress has been made in the separate analysis of the effects of context and of latency. We feel that this is a serious shortcoming of the literature, as well as an explanation for the discrepancy in elicited cancer premium values. Essentially, if delayed fatality risks are indeed discounted by respondents in valuation studies, then each point estimate of the cancer VSL is not generalizable away from the specific context and delay for which the relativity was elicited. However, if the effective discount rate can be disentangled from the (time invariant) context effects, then it would be possible to apply the valuation to cancer scenarios with different latency periods. The 'dread' and latency effects are well documented as discussed, but the literature lacks a framework for their specific and simultaneous analysis. To address this, we propose the following structural relationship:

$$
\frac{C_{T}}{R_{t}}=\frac{(1+x)}{(1+r)^{(T-t)}}
$$

In which $\mathrm{C}_{\mathrm{T}}$ is the $\mathrm{VSL}_{\mathrm{CANCER}}$ when the cancer fatality would occur at time $\mathrm{T}, \mathrm{R}_{\mathrm{t}}$ is the VSL for risks of road accident fatalities which would occur at time $t(t \leq T),(1+\mathrm{x})$ is a scalar which captures the effects of the context of cancer, including dread and morbidity effects; and $\mathrm{r}$ is the discount rate which is assumed to be exponential for tractability. ${ }^{6}$ We apply discounting to all periods until fatality would occur, and as such both the latency and morbidity periods contribute to the discount factor. In this framework, we capture the major qualitative differences between cancer fatality and road accident fatality risk valuations. We can use the framework to elicit estimates of the context premium $(1+\mathrm{x})$ and the discount rate $(\mathrm{r})$.

In what follows, we present a survey that supports the proposed $\mathrm{C}_{\mathrm{T}} \mathrm{R}_{\mathrm{t}}$ relationship by confirming the directional effects of context and latency in a controlled survey design. We verify that the context of cancer increases the relative size of the VSL, and that longer latency periods reduce it, ceteris paribus. We estimate the size of the context and latency effects using regression analysis. The data are then used to investigate the overall relativities between the VSL for cancer with different latency and morbidity characteristics, and that for instantaneous road accident fatalities.

\section{Methods}

\subsection{Risk-Risk trade-offs}

The Risk-Risk trade-off method, developed by Viscusi et al. (1991), and extended in Magat et al. (1996) and Van Houtven et al. (2008), allows respondents to compare risks

\footnotetext{
${ }^{6}$ The relationship can be adapted for hyperbolic and other discounting hypotheses simply by adjusting the denominator. 
of fatality in one cause with risks of fatality in another, thereby avoiding the need for complex money-risk trades that are necessary for standard contingent valuation. It also reduces the likelihood of scope insensitivity problems as documented in Jones-Lee et al. (1985) or Beattie et al. (1998). The method provides a measure of the strength of preference for avoiding specified risks, and has been shown by Van Houtven et al. (2008) to be viable in investigating preferences over cancer fatalities.

Following Viscusi et al. (1991); Magat et al. (1996), and Van Houtven et al. (2008) this model incorporates three possibilities, death in a road accident $(D)$, death by cancer $(C)$ and full health $(H)$ resulting from avoidance of death in a road accident or by cancer. These have corresponding lifetime utilities that depend on both the cause of death and on wealth, $w$, represented by $U(D, w), U(C, w)$ and $U(H, w)$; and respective probabilities $\pi_{D}, \pi_{C}$ and $\left(1-\pi_{D}-\pi_{C}\right)$.

Therefore expected lifetime utility is given by

$$
E(U)=\pi_{D} U(D, w)+\pi_{C} U(C, w)+\left(1-\pi_{D}-\pi_{C}\right) U(H, w)
$$

Now consider a choice between two options, A and B, where the probabilities of road accident and cancer fatality differ. These are denoted $\pi_{D}^{A}, \pi_{D}^{B}, \pi_{C}^{A}$, and $\pi_{C}^{B}$.

For an expected utility maximiser, indifference between options A and B implies:

$$
\begin{aligned}
& \pi_{D}^{A} U(D, w)+\pi_{C}^{A} U(C, w)+\left(1-\pi_{D}^{A}-\pi_{C}^{A}\right) U(H, w) \\
& =\pi_{D}^{B} U(D, w)+\pi_{C}^{B} U(C, w)+\left(1-\pi_{D}^{B}-\pi_{C}^{B}\right) U(H, w)
\end{aligned}
$$

To recast the analysis in terms of the VSL, recognise that the standard VSL is defined as the marginal rate of substitution between wealth and risk of an instant road accident fatality. Similarly, the $\mathrm{VSL}_{\mathrm{CANCER}}$ is the marginal rate of substitution between wealth and risk of cancer fatality. Setting U(D) equal to zero (with no loss of generality) and differentiating Eq. (2) with respect to $\pi_{D}, \pi_{C}$, and wealth, Van Houtven et al. (2008) show that the relativity between VSL $_{\text {CANCER }}$ and the standard VSL is

$$
\frac{V S L_{\mathrm{CANCER}}}{V S L}=1-\frac{U(C, w)}{U(H, w)}
$$

So that with $U(C, w)<0<U(H, w)$ then $\frac{V S L_{\text {CANCER }}}{V S L}>1$, while with $0<U(C, w)$ $<U(H, w)$ then $\frac{V S L_{\text {CANCER }}}{V S L}<1$. That is, the framework does not constrain $U(C, w)$ to be greater than or smaller than $U(D, w)$.

Of course, it is not possible to directly observe utilities from Risk-Risk survey data. Instead, we observe the relative sizes of risk changes that make respondents indifferent between options A and B. From Eq. (3), with $U(D, w)=0$, then

$$
U(C, w)=\left[1-\left(\frac{\pi_{D}^{B}-\pi_{D}^{A}}{\pi_{C}^{A}-\pi_{C}^{B}}\right)\right] U(H, w)
$$


Rearranging, and in combination with Eq. (4),

$$
\frac{V S L_{\mathrm{CANCER}}}{V S L}=1-\frac{U(C, w)}{U(H, w)}=\frac{\pi_{D}^{B}-\pi_{D}^{A}}{\pi_{C}^{A}-\pi_{C}^{B}}
$$

By observing the relative sizes of $\pi_{D}$ and $\pi_{C}$ that make respondents indifferent between options $\mathrm{A}$ and $\mathrm{B}$, one can therefore determine the relative size of the value of statistical life for one cause compared to another. This result, previously demonstrated in Van Houtven et al. (2008), makes intuitive sense in that if the change in cancer risk between options A and B is precisely offset for the respondent by the change in road accident risk, then the ratio of the respondent's MRS of wealth for risk (i.e. the ratio of his/her VSLs) must be equal to the inverse of the ratio of the risk changes. More

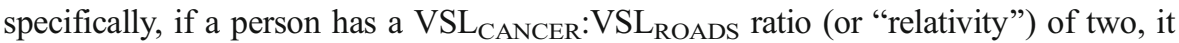
implies they would be indifferent between an increase (decrease) in their risk of road accidents that is twice as large as the increase (decrease) in their risk of cancer fatality. Further, the relative size of the VSL for cancer and road accidents, and the relative size of the risk differences that render the respondent indifferent, reflect the proportional utility loss in the case of cancer $\left(\frac{U(H, w)-U(C, w)}{U(H, w)}\right)$.

\subsection{Survey}

We administered a Risk-Risk trade-off survey to capture the relative VSL for roads and cancer with different latency periods. The survey was administered in a small group setting, as part of a wider interview protocol with three stages. ${ }^{7}$ All answers were elicited on an individual basis.

The first stage comprised a combination of learning exercises designed to familiarise respondents with the type of questions and elicitation procedures used in Risk-Risk trade-off studies. There were also some structured questions and open-ended discussions to check for comprehension of key concepts such as risk and timing of death. We emphasised the cost to the individual of accepting a bigger risk increase in their less feared cause by reminding them about the resulting increase in their total risk of death. Respondents were reminded that this cost could be avoided by switching to the smaller increase in the risk of their more feared cause. ${ }^{8}$ The later phase introduced the two risk contexts to be considered in the main survey - death in a road accident and death by cancer. We also introduced the pictorial representations that would be used to describe the scenarios, so-called "Profiles" (Fig. 1) as well as the symptoms associated with a typical cancer case. The symptoms were described in as much detail as possible,

\footnotetext{
${ }^{7}$ Extracts are provided in the appendix, and the full interview protocol and questionnaire are available on request.

${ }^{8}$ In piloting, the Risk-Risk trade-off questions used risk decreases, but this proved too cognitively complex for most respondents and generated implausibly large relativities. We changed to the domain of risk increases, using the scenario of spending reductions in the government's safety budget. Under Expected Utility theory, marginal increases and decreases in risk can be assumed to have equal magnitude because of the assumption that linearity holds at the margin. The proposed analysis is unaffected by the choice of risk increases or risk reductions. Despite using risk increases the elicited relativities were still very large. As a result we developed a pie-diagram that illustrated the direct "cost" to the respondent of taking the bigger risk increase; specifically, the larger increase in total risk of death.
} 


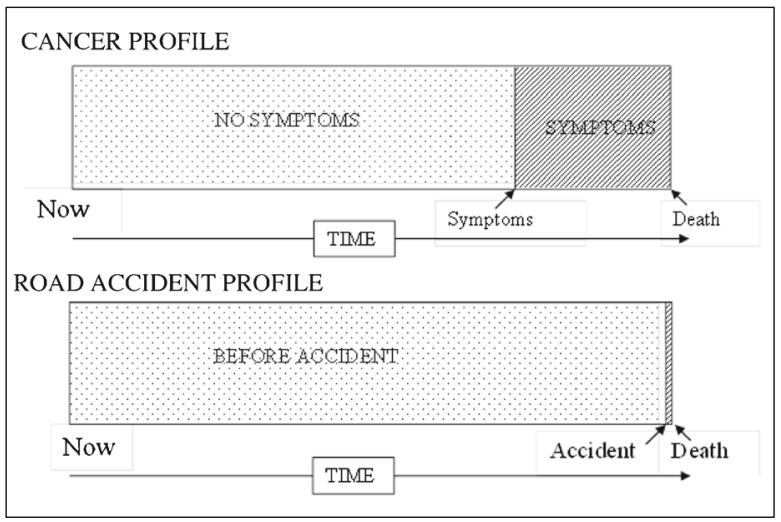

Fig. 1 Profiles used to describe the cancer and road accident fatality scenarios

without losing the generality of the scenario. An extract from the experimenter's script describing the cancer symptoms, along with other aspects of the cancer scenarios, is provided in the appendix. We specified that the cancers were not behaviour-related (for example, not smoking-related), instead being due to exposure to environmental or workplace causes.

The cancers described had three key timing features: time of exposure, time of symptom onset, and time of death. These then determined the latency and morbidity periods. ${ }^{9}$ Notice that we never explicitly referred to "latency" or "morbidity" in the experiment, instead referring to "the time until you would become ill", "the time spent ill" and "the time of your death", to simplify the explanation (see the appendix for some example text). In every case, the individual would be exposed to the carcinogen during the coming year; however, the latency and morbidity periods varied across scenarios. So, for example, cancer fatality in 10 years refers to exposure during the coming year, onset of symptoms after 9 years, and death 1 year after that, 10 years from now.

An important distinction is the time of exposure (in our questions, this was always during the coming year, with any associated monetary costs or savings happening now) and the time that the cancer would physically manifest itself (encompassing illness and death).

The second stage of the session comprised the ten Risk-Risk trade-off questions. ${ }^{10}$ These are summarised in Table 2, along with a brief description of the purpose of each question. In every case, both baselines were presented as 1000 in 60 million. The denominator of 60 million was chosen because it is the size of the UK population, and respondents were informed of this to help them to put the size of the risks into meaningful context. We chose 1000 as the numerator because the average road accident

\footnotetext{
${ }^{9}$ We vary duration of illness as opposed to severity because duration is an objectively increasing measure of illness. The analysis could usefully be extended to consider severity of symptoms in future.

${ }^{10}$ All respondents answered all ten questions and as such there exists a danger of "anchoring" or "learning" leading to non-independent responses, as well as fatigue. To ensure that the relativities to be used in the calculation of the parameter values $\mathrm{r}$ and $(1+\mathrm{x})$ were free from these particular biases, we chose to ask the 'overall' questions (Q1-Q3) first for every respondent. We decided against random ordering of the remaining questions, instead keeping a logical ordering of question sets, to avoid confusing respondents by frequently switching contexts, duration etc. We did, however, reverse the order of questions within sections to minimize any anchoring or learning effects between questions where direct comparisons would be made.
} 
Table 2 Risk-Risk questions

\begin{tabular}{|c|c|c|c|}
\hline Purpose & Question & Explanation & Code \\
\hline \multirow[t]{3}{*}{ Premium* } & Q1 & Road death in the coming year vs cancer death in 10 years & $\mathrm{C}_{10}: \mathrm{R}_{1}$ \\
\hline & Q2 & Road death the year after next vs cancer death in 10 years & $\mathrm{C}_{10}: \mathrm{R}_{2}$ \\
\hline & Q3 & Road death the year after next vs cancer death in 25 years & $\mathrm{C}_{25}: \mathrm{R}_{2}$ \\
\hline \multirow[t]{2}{*}{ Context } & Q4 & Road death the year after next vs cancer death year after next & $\mathrm{C}_{2}: \mathrm{R}_{2}$ \\
\hline & Q5 & Road death in 10 years vs cancer death in 10 years & $\mathrm{C}_{10}: \mathrm{R}_{10}$ \\
\hline \multirow[t]{2}{*}{ Latency } & Q6 & Cancer death year after next vs cancer death in 10 years & $\mathrm{C}_{2}: \mathrm{C}_{10}$ \\
\hline & Q7 & Cancer death year after next vs cancer death in 25 years & $\mathrm{C}_{2}: \mathrm{C}_{25}$ \\
\hline \multirow[t]{2}{*}{ Morbidity } & Q8 & $\begin{array}{l}\text { Cancer death in } 10 \text { years ( } 6 \text { months' illness prior to death) vs } \\
\text { Cancer death in } 10 \text { years ( } 1 \text { year's illness prior to death) }\end{array}$ & $\begin{array}{l}\mathrm{C}_{10}[12 \mathrm{~m}]: \\
\mathrm{C}_{10}[6 \mathrm{~m}]\end{array}$ \\
\hline & Q9 & $\begin{array}{l}\text { Cancer death in } 10 \text { years ( } 6 \text { months' illness prior to death) vs } \\
\text { Cancer death in } 10 \text { years ( } 3 \text { years' illness prior to death) }\end{array}$ & $\begin{array}{l}\mathrm{C}_{10}[36 \mathrm{~m}]: \\
\mathrm{C}_{10}[6 \mathrm{~m}]\end{array}$ \\
\hline Labelling & Q10 & $\begin{array}{l}\text { Road death in } 10 \text { years (1-2 weeks' illness prior to death) vs } \\
\text { Cancer death in } 10 \text { years (1-2 weeks' illness prior to death) }\end{array}$ & $\mathrm{C}_{10}[2 \mathrm{w}]: \mathrm{R}_{10}[2 \mathrm{w}]$ \\
\hline
\end{tabular}

*Premium questions incorporate differences in latency, morbidity and context in an overall comparison

risk in the UK can be rounded up to 1000 in 60 million. ${ }^{11}$ In addition, the attributes of the cancers presented (typical latency and typical morbidity period) mean that we can construct groups of cancers with those properties, and cancers can be chosen such that the group's overall baseline is close to 1000 in 60 million. ${ }^{12}$

Q1-Q3 most closely match those typically asked in the literature. The comparison was between road accidents during the coming year (in Q1) or during the year after next (in Q2-3) and cancers with 9 or 24 years of latency, one year of morbidity and then fatality after a total of 10 or 25 years. These latencies were chosen to provide information for a credible range of latency periods for a variety of cancers. Throughout, we refer to this latent cancer to current roads relativity as the "overall premium". The relativity was elicited for the risks of cancer fatality in 10 years relative to both road fatality risks in the current year and also for road fatality risks the year after next, in case a strong 'present bias' was prevalent in responses to Q1. If preferences were presentbiased, then the weight on avoiding a risk increase in the coming year would be large relative to that for future years, generating a potential for respondents to overstate the acceptable future risk to avoid an increase in current period risk, however small. For notation, these questions will be referred to as $\mathrm{C}_{10} \mathrm{R}_{1}, \mathrm{C}_{10} \mathrm{R}_{2}$ and $\mathrm{C}_{25} \mathrm{R}_{2}$ where the letter denotes the cause of death (context) and the number denotes the number of years until fatality. In the cancer case, this incorporates T-1 years of latency and a fixed 1 year morbidity period. For road accidents, we described the morbidity period as lasting "minutes or hours". These questions provide information about the level of the overall cancer premium for a given latency and morbidity.

A multiple-list elicitation format was adopted. The method and materials are similar to those explained in Chilton et al. (2006)). The method is analogous to a payment card

\footnotetext{
${ }^{11}$ The reason for equalising the baselines is that very early in the piloting stage, it became apparent that baseline impacts would overwhelm any dread or latency effects of the sort we aimed to elicit.

12 Derived from the HSE Dataset "Input data for cancer registrations".
} 
procedure in the context of a WTP question and shares its associated advantages and disadvantages. In each question, respondents were asked to choose between increases in their risk of dying by one of the two causes if the increase in risk was 50 in 60 million in both cases (or to express indifference). Then they were asked to choose between the two risk increases in each row, and hence to reveal what level of risk increase would make them "switch over" to the smaller increase in the other risk. This reveals a narrow range for their indifference point in the table. ${ }^{13}$ For example, if a respondent chose to accept the increase in their road accident fatality risk, they received an answer sheet in which the road accident risk was increased in each row. ${ }^{14} \mathrm{We}$ analyse the first row in which they would prefer to take the smaller risk increase (50 in 60 million) in the other, more feared cause of fatality. This switching point forms the basis for the calculation of marginal rates of substitution described in the next section. If a respondent reached the bottom of the table and had not yet switched from their most preferred risk increase, they were asked to indicate the highest risk increase that they would be prepared to incur before switching. This ensures that data are available to calculate relativities for respondents with "extreme" preferences. ${ }^{15}$

Subsequent to questions that elicit the overall premium (Q1-3), respondents answered seven further questions designed to verify the structural relationship proposed in Eq. (1). These questions also allow us to run some individual-level scope tests. Q4-5 estimate the significance of the context premium, since the timing of death is held constant in each of the questions. Morbidity is again fixed at 12 months for the cancer and "minutes or hours" for the road accident. These questions (and resulting relativities) have the labels $\mathrm{C}_{2} \mathrm{R}_{2}$ and $\mathrm{C}_{10} \mathrm{R}_{10}$ following the convention outlined above. Q6-7 $\left(\mathrm{C}_{2} \mathrm{C}_{10}\right.$ and $\left.\mathrm{C}_{2} \mathrm{C}_{25}\right)$ capture latency effects by comparing cancer fatalities now and in the future, holding context and morbidity constant. Q8-9 capture morbidity effects by varying the period that the individual is ill prior to death, but holding the timing of death constant. The notation is extended to include the number of months of illness in square brackets (see Table 2). Q4-9 provide some validity to the context premium results, as well as generate grounds for future investigation of what might drive the context premium. Finally, Q10 is designed to rule in or rule out any 'labelling' effects i.e. whether the word "cancer" triggers a stronger preference for avoiding this risk, even when the morbidity period is the same in both cases.

\footnotetext{
${ }^{13} \mathrm{We}$ analyse the mid-point of this range as their indifference point.

${ }^{14}$ A potential confound arises because respondents may be able to deduce when others expressed indifference between the initial risk increase because they would not receive a second answer sheet. It is unclear which direction this might bias respondents (if any), but it may induce respondents to herd towards or away from stating indifference during the course of the session. We checked the number of 'indifference' answers per question order and found no evidence that the number or proportion of 'indifference' responses increased or decreased as the survey progressed. We note that there were a small minority of indifference responses, and for Q1-9 they made up just 7.14 \% of the total responses. We also checked for a peak at the first row of switching which could indicate people being reluctant to publicly admit indifference between the two risks. This was not evident.

${ }^{15}$ To check for any obvious biases in our responses due to the use of this elicitation mechanism, we ran some standard checks. Only one respondent switched multiple times in our survey. Plotting the frequency distribution provided no strong evidence to suggest that responses were clustered around the middle of the table. Of the responses from within the body of the table, the modal switch-point was in row 6 of 9. A large proportion of respondents switched just below the table, indicating values of up to 800 in 60 million. A similar proportion indicated indifference. This suggests that respondents did not feel constrained by the endpoints of the table.
} 
The final stage contained questions designed to elicit respondents' attitudes to financial risk; time preferences; and health risk preferences, all of which could help to explain respondents' R-R trade-offs. Demographic questions concluded the session, which lasted about one and three quarter hours.

\section{Results}

One hundred fifty-seven residents of the City of Newcastle Upon Tyne, UK were interviewed in January 2012. The respondents were aged between 30 and 50 and broadly representative of the population of the North East of England in that age cohort, although unemployed people were slightly overrepresented. Table 3 displays mean values with standard errors in parentheses.

We present the central tendency results in Table 4, using the geometric mean for each relativity since this central tendency measure is arguably the best suited to the analysis of ratio data, avoiding the bias associated with the arithmetic mean ${ }^{16}$ and displaying more sensitivity than the median. The results are not qualitatively affected by this choice. The table shows the number of responses removed during data cleaning, where protests are taken out of the dataset. These are defined as responses stating that no amount of risk increase would induce them to switch, probabilities greater than 1 , or in one case, a response indicating multiple switchpoints. Unanswered questions are also included. $64.2 \%$ of respondents provided an answer to every question in the survey, and only $3.1 \%$ gave a protest response in all ten questions.

On reviewing the remaining data we found a proportion of high-end outliers in the initial dataset, and we trimmed the dataset as follows. First, we set a threshold above which we felt answers were unreasonably high. We chose to exclude responses of 30 million in 60 million and above (i.e. any response saying "I would switch if the increase in the risk of my less feared cause rose to 30 million in 60 million" or more). Remember that the risk of their initially less preferred cause was only 50 in 60 million, and so a response of 30 million or more suggests that respondents considered their most feared cause to be 600,000 times as bad as their less feared cause. ${ }^{17}$

We trim on an individual question basis as opposed to a respondent basis, by removing specific responses that fell above the thresholds. Between 0 and 14 responses were trimmed from each question. $79.6 \%$ of respondents did not have any responses trimmed from the dataset, $5.0 \%$ had just 1 response removed, and no one had all ten responses trimmed, which suggests that a proportion of individuals made errors or expressed very strong preferences in a subset of questions. This behaviour was most common in the latency questions (comparing cancer at two different times), perhaps reflecting lexicographic preferences for delaying the fatality.

After trimming, the remaining per-question sample size ranges are from 117 to 139 . We present the untrimmed and trimmed responses in Table 4. However we focus on the trimmed dataset for the purpose of commentary.

\footnotetext{
${ }^{16}$ In which the cause of death in the numerator is effectively over-weighted.

${ }^{17}$ Some respondents were prepared to accept a risk increase of 50 million in 60 million, indicating a premium of 1 million to 1 . 
Table 3 Sample demographics

\begin{tabular}{ll}
\hline Characteristic & Sample $(n=157)$ \\
\hline Female (\%) & 52.6 \\
Age (years) & 39 \\
$\quad$ Mean & $(5.75)$ \\
$($ s.e. $)$ & 37.5 \\
Median & \\
Household size & 3.27 \\
Mean & $(1.36)$ \\
$\quad$ s.e.) & \\
Number of children in household & 1.38 \\
Mean & $(1.19)$ \\
(s.e.) & 23.2 \\
Households with children under 6 years old (\%) & \\
Household income (GBP/month) & 2685.52 \\
Mean & $(2074.88)$ \\
$($ s.e.) & 2500 \\
Median & 39.7 \\
Rented home (\%) & 48.0 \\
Further education (\%) & 73.5 \\
Experience of cancer (self or close friend or family member) (\%) & 50.3 \\
Experience of road accidents (self or close friend or family member) (\%) & \\
\hline
\end{tabular}

\subsection{Validating the $C_{T} R_{t}$ relationship}

The central tendencies of the relativities provide evidence in support of the $\mathrm{C}_{\mathrm{T}} \mathrm{R}_{\mathrm{t}}$ relationship proposed in Eq. (1). We find a positive effect of context and morbidity, while latency reduces the relativity. We consider each result in turn. After validating that the effects appear to hold in general, we run some internal scope tests, checking that the patterns of response between question blocks is consistent for the majority of respondents.

\subsubsection{Context and morbidity}

By holding time constant, and comparing road accident and cancer risks where the fatality would occur at the same time - after 2 years in Q4 and 10 years in Q5, the context questions provide information on how respondents view contemporaneous risks of cancer and road accident fatalities. Notice that the latency period for cancer is t-1 years, allowing the 1 year morbidity period to occur and the time of fatality to be equal in the cancer and road accident cases. This avoids the confound between length of life and quality of life that is sometimes encountered when morbidity is presented as an "add-on".

A strong preference is indicated for avoiding cancer risk increases as opposed to road accident risk increases in both questions $4\left(\mathrm{C}_{2}: \mathrm{R}_{2}\right)$ and $5\left(\mathrm{C}_{10}: \mathrm{R}_{10}\right)$. Twelve months of illness or morbidity is included in the cancer. 
Table 4 Central tendency measures of relative size of VSLs compared in each question

\begin{tabular}{|c|c|c|c|c|}
\hline \multirow[t]{2}{*}{ Question } & \multicolumn{2}{|c|}{ Untrimmed data } & \multicolumn{2}{|c|}{ Trimmed data } \\
\hline & $n$ & $\begin{array}{l}\text { Geometric mean } \\
{[95 \% \text { confidence interval }]}\end{array}$ & $n$ & $\begin{array}{l}\text { Geometric mean } \\
{[95 \% \text { confidence interval }]}\end{array}$ \\
\hline $1\left(\mathrm{C}_{10} \mathrm{R}_{1}\right)$ & 138 & $\begin{array}{l}1.230 \\
{[0.597,3.631]}\end{array}$ & 133 & $\begin{array}{l}1.116 \\
{[0.576,2.161]}\end{array}$ \\
\hline $2\left(\mathrm{C}_{10} \mathrm{R}_{2}\right)$ & 136 & $\begin{array}{l}0.556 \\
{[0.236,1.314]}\end{array}$ & 128 & $\begin{array}{l}0.814 \\
{[0.403,1.643]}\end{array}$ \\
\hline $3\left(\mathrm{C}_{25} \mathrm{R}_{2}\right)$ & 136 & $\begin{array}{l}0.228 \\
{[0.095,0.545]}\end{array}$ & 127 & $\begin{array}{l}0.280 \\
{[0.139,0.566]}\end{array}$ \\
\hline $4\left(\mathrm{C}_{2} \mathrm{R}_{2}\right)$ & 132 & $\begin{array}{l}11.269 \\
{[5.048,25.156]}\end{array}$ & 125 & $\begin{array}{l}9.340 \\
{[4.806,18.151]}\end{array}$ \\
\hline $5\left(\mathrm{C}_{10} \mathrm{R}_{10}\right)$ & 135 & $\begin{array}{l}9.697 \\
{[4.200,22.388]}\end{array}$ & 128 & $\begin{array}{l}9.818 \\
{[4.905,19.652]}\end{array}$ \\
\hline $6\left(\mathrm{C}_{2} \mathrm{C}_{10}\right)$ & 135 & $\begin{array}{l}52.176 \\
{[25.783,105.587]}\end{array}$ & 124 & $\begin{array}{l}22.297 \\
{[12.806,38.822]}\end{array}$ \\
\hline $7\left(\mathrm{C}_{2} \mathrm{C}_{25}\right)$ & 131 & $\begin{array}{l}79.016 \\
{[37.630,165.920]}\end{array}$ & 117 & $\begin{array}{l}26.356 \\
{[15.097,46.009]}\end{array}$ \\
\hline $8\left(\mathrm{C}_{10}[12] \mathrm{C}_{10}[6]\right)$ & 139 & $\begin{array}{l}9.118 \\
{[4.420,18.808]}\end{array}$ & 133 & $\begin{array}{l}8.215 \\
{[4.534,14.89]}\end{array}$ \\
\hline $9\left(\mathrm{C}_{10}[36] \mathrm{C}_{10}[6]\right)$ & 130 & $\begin{array}{l}27.752 \\
{[12.946,59.490]}\end{array}$ & 124 & $\begin{array}{l}17.051 \\
{[8.552,33.994]}\end{array}$ \\
\hline $10\left(\mathrm{C}_{10}[2 \mathrm{w}] \mathrm{R}_{10}[2 \mathrm{w}]\right)$ & 139 & $\begin{array}{l}0.943 \\
{[0.614,1.447]}\end{array}$ & 139 & $\begin{array}{l}0.943 \\
{[0.614,1.447]}\end{array}$ \\
\hline
\end{tabular}

The relativities from Q4 and Q5 $\left(\mathrm{C}_{2}: \mathrm{R}_{2}\right.$ and $\left.\mathrm{C}_{10}: \mathrm{R}_{10}\right)$ are not significantly different to one another. This suggests that for cancer risk increases (with 1 year of illness preceding death) compared to contemporaneous road accident risk increases, the relativity is not affected by when the risk increases would occur. This implies that risks of both causes of death are discounted by the individual at the same rate, and that discounting applies to morbidity and death combined in the cancer case.

Turning to morbidity, the responses to Q8 and Q9 show less illness is preferred to more and as the amount of illness increases, the relativity increases at a decreasing rate. The premia for both questions are significantly different than 1 .

To test whether morbidity is the only driver of the context effect, a final roads:cancer comparison question $\left(\mathrm{Q} 10, \mathrm{C}_{10}[2 \mathrm{w}]: \mathrm{R}_{10}[2 \mathrm{w}]\right)$ is included, in which the scenarios both include two weeks of illness prior to fatality ten years from now. In every case, using every measure of central tendency and at every level of trimming, the ratio is insignificantly different to one, suggesting that the label of cancer by itself does not give rise to a cancer premium. ${ }^{18}$

Overall, the context and morbidity results support the assumptions that context influences the VSL and that in the cancer to roads comparison the cancer context

\footnotetext{
18 This suggests that the insights about latency and morbidity might generalise to other latent fatalities preceded by morbidity, although empirical work will be required to verify this.
} 
(including a morbidity period) increases the VSL relative to the roads VSL. This supports the numerator of the relationship proposed in Eq. (1).

\subsubsection{Latency}

The latency questions compared death from cancer during the year after next with death from cancer 10 or 25 years into the future. The person would be exposed today, have a latency period of 1,9 or 24 years with no noticeable change to their health, then 12 months' morbidity, and then they would die.

The central tendencies show a strong preference to avoid cancer the year after next compared to cancer 10 or 25 years from now (Q6 and Q7), with the relativities for both of these questions significantly exceeding 1 . This lends support to the denominator of the relationship proposed in Eq. (1), by demonstrating that respondents are on average willing to accept a larger fatality risk later to avoid a risk increase now, so that latency reduces the relative size of the implied VSL $_{\text {CANCER. }}$.

\subsection{Cancer premium}

The insights about latency, morbidity and context discussed so far were generated by questions isolating the dimension of interest, providing easily interpreted findings. However, surveys will more typically compare scenarios that vary these dimensions simultaneously, and this was replicated in our survey questions 1-3 which compare risks of road accident fatalities one or two years from now to risks of cancer fatalities after 10 or 25 years with one year of morbidity. If the offsetting effects proposed in the relationship in Eq. (1) are correct, then the elicited relativities from Q1-3 would be expected to be closer to 1 than either the latency or context question relativities. This is indeed what we find in our data.

The cancer to roads relativity is insignificantly different to one for both Q1 (cancer fatality in 10 years versus a roads fatality in 1 year i.e. $\left.C_{10} R_{1}\right)$ and Q2 $\left(C_{10} R_{2}\right)$ and is significantly lower than one for question three $\left(\mathrm{C}_{25} \mathrm{R}_{2}\right)$. Respondents appeared to view the difference between "during the coming year" and "during the year after next" as insignificant because the relativities for $C_{10} R_{1}$ and $C_{10} R_{2}$ are statistically indistinguishable. Q3's $\left(\mathrm{C}_{25}: \mathrm{R}_{2}\right)$ relativity is significantly smaller than the relativity for $\mathrm{Q} 2\left(\mathrm{C}_{10}: \mathrm{R}_{2}\right)$. This suggests that the difference between risks of death from cancer 10 years from now and risks of death from cancer 25 years from now is perceived to be significant, when compared to risks of death in a road accident the year after next. The 24 year latency period is perceived as significantly preferable to the 9 year latency. This is further evidence that latency appears to reduce the VSL for cancer.

\subsection{Internal scope tests}

The above sections validated the prior assumptions about the direction of preference for each block of questions, finding that on an aggregate level the sample responses conform to theoretical expectations. Now, on an individual level, we can identify the extent to which each respondent's responses conform to the theoretically predicted patterns, to investigate their internal consistency (Table 5). 
Table 5 Results of internal consistency and scope tests: Proportions of individuals violating quality checks of the data

\begin{tabular}{|c|c|c|c|c|}
\hline & Q2-3 & Q4-5 & Q6-7 & Q8-9 \\
\hline Missing & $37 / 159=23 \%$ & $38 / 159=24 \%$ & $47 / 159=30 \%$ & $38 / 159=24 \%$ \\
\hline Inconsistent $^{a}$ & No prediction & $4 / 121=3 \%$ & $1 / 112=1 \%$ & $9 / 121=7 \%$ \\
\hline $\begin{array}{l}\text { Absolute difference from } 1 \text { is contrary } \\
\text { to prediction }{ }^{b}\end{array}$ & No prediction & $16 / 115=14 \%$ & $3 / 111=3 \%$ & $10 / 112=9 \%$ \\
\hline $\begin{array}{l}\text { Direction of change in relativity between } \\
\text { questions is contrary to prediction }{ }^{\text {a }}\end{array}$ & $12 / 122=10 \%$ & No Prediction & $12 / 112=11 \%$ & $17 / 121=14 \%$ \\
\hline Same response in both questions ${ }^{a}$ & $42 / 122=34 \%$ & $67 / 121=55 \%$ & $65 / 112=58 \%$ & $48 / 121=40 \%$ \\
\hline
\end{tabular}

$\mathrm{a}=$ percentage of non-missing responses, ${ }^{\mathrm{b}}=$ percentage of non-missing and consistent responses

\subsubsection{Q4-5: Context}

For $24 \%$ of respondents, one or both responses are missing. In questions 4 and 5 , the respondent is consistent if they have the same initial preference between cancer and road accident risk increases between the questions. Only 3\% of individuals have inconsistent responses. Also, cancer might be considered worse than car because of morbidity and dread effects. Just $14 \%$ of respondents prefer a road accident increase when time of death is held constant. However, arguably this should not be interpreted as a test of response quality, but instead as a result in its own right.

\subsubsection{Q6-7 Latency}

We expect respondents to prefer to avoid the earlier risk increase in both Q6 and Q7. Of the $70 \%$ of respondents who do not have missing responses, just three respondents (3\%) violate this prediction, and only 1 flips between preferring earlier and later. As a scope test, we expect the respondents to more strongly prefer to avoid the earlier risk increase in Q7 than in Q6, because of the additional 15 years' latency. Of course, if different future life years are valued differently (for example because of the demands of childcare or perceptions of wellbeing in different stages of life) this may not be true, but we would expect a majority of respondents to conform. In fact, just $11 \%$ of respondents have a smaller relativity in Q6 than in Q7, but 58\% show no difference between the questions, which could indicate a lack of sensitivity to scope for a majority of this sample. A plausible alternative explanation is that the difference between 10 and 25 years may not have been very important to respondents, compared to the difference between "this year" and "later". As such, and if respondents tended to give round number estimates, this might drive some of the lack of sensitivity.

\subsubsection{Q2-3 Context and latency combined}

We impose no predictions about whether the relativities in Q2-3 are greater or less than 1 , because of the offset between context and latency effects. However, due to the difference in latency periods we would expect a greater relativity in Q3 than in Q2. This 
holds for the majority of respondents, though $34 \%$ are insensitive to the change in latency period in Q2-Q3, suggesting that the effect of timing is different when the types of fatality differ. $10 \%$ express a smaller relativity in Q3. One or both responses are missing for $23 \%$ of the sample.

\subsubsection{Q8-9 Morbidity}

We expect respondents to prefer shorter to longer morbidity. We do not encounter any confound between length and quality of life because in our design length of life is held constant at 10 years. The difference is entirely the length of morbidity (and corresponding reduction in healthy years of life). A preference for longer morbidity could reflect that it gives the person longer to prepare for their death, but in general we expect a higher relativity in Q9 than in Q8, with both relativities greater than 1. Our results support this to a large extent: just $9 \%$ prefer longer morbidity to smaller in both questions (7\% are inconsistent), and just $14 \%$ have a smaller relativity in Q9. However, $40 \%$ are insensitive to the difference between the questions.

\subsection{Regression analysis}

The central tendencies lend support to the $C_{T} R_{t}$ relationship proposed in Eq. (1). There seems to be a strong effect of both context and latency when they are isolated in survey questions. However, analysis of the central tendencies alone ignores any influence of demographics, and can by definition only incorporate comparisons across sample averages. To address both of these issues we pool the data from all questions in the survey and run overall regressions for the full sample. The dependent variable is the natural $\log$ of the relativity (regardless of the question that elicited it) and the effects of context, latency, morbidity and labelling are included as explanatory variables. We estimate a series of OLS regressions of the pooled logged relativities against question characteristics and demographics. We cluster on the individual to account for the elicitation of multiple relativities per respondent. We also present fixed effects regression results, which control for individual heterogeneity. The results are included in Table 6, which gives each coefficient with its standard error in brackets.

Model (1) is an OLS regression including context, time until death and morbidity differences between the questions that underpin the dependent variable (the log of the relativity) and observable demographic characteristics. The 'time until death' variable is included instead of latency, because it facilitates the comparison between the crosscause fatalities (Cancer:Roads) and the within-cause fatalities (Cancer:Cancer), as it avoids the confound by which the same latency difference could give different life expectancies depending on the length of the morbidity period. Time until death and morbidity are the key drivers of the relativity, and although the cancer context dummy has a positive coefficient, it is insignificant. This suggests that morbidity drives the context effect for cancer, a result supported by the lack of labelling premium that was found in question $10\left(\mathrm{C}_{10}[2 \mathrm{w}] \mathrm{R}_{10}[2 \mathrm{w}]\right)$. Model (2) uses the insight from the central tendencies that the longer latency and morbidity periods do not appear to be linearly reflected in their relative severities. That is, there might be a non-linear relationship between morbidity, time until death and the overall relativities. We explore this by including squared terms on each of these variables, and these effects are significant in 
Table 6 Regression analysis of logged relativities (pooled) against question characteristics and demographics

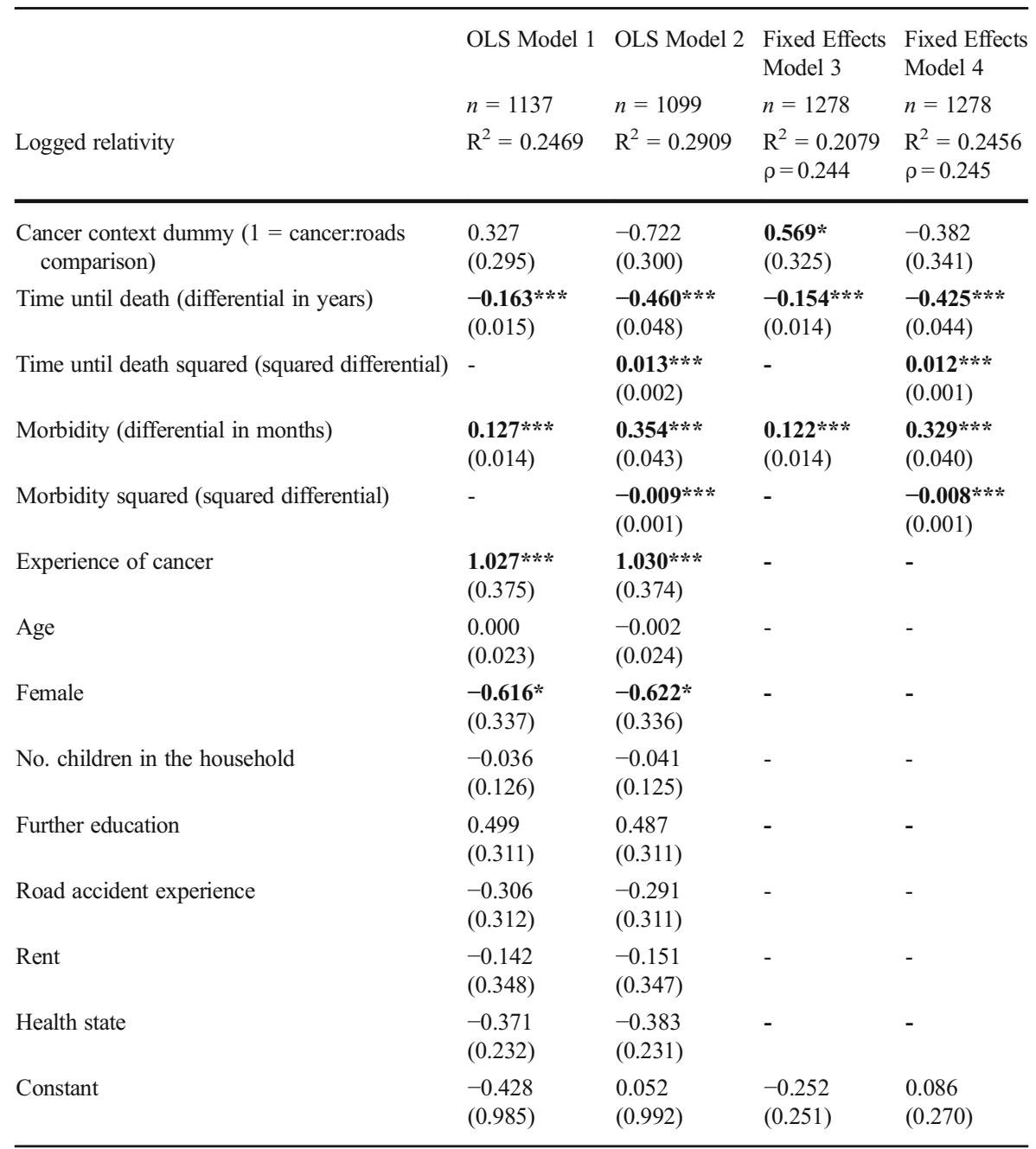

Asterisks denote p-statistic thresholds with $*=p<0.1, * *=p<0.05$ and $* * *=p<0.01$.

the expected directions: additional differences between the times until death will decrease the relativities at a decreasing rate, while additional morbidity differences will increase the relativity at a decreasing rate. The overall fit of this model is high, suggesting that diminishing effects of morbidity and latency are important in explaining the VSL for cancer.

Demographic variables are largely insignificant, although female respondents are slightly more willing to take risks of the numerator causes (cancer in the cross-context questions, or later fatality in the within-cancer latency questions) than men, while those with experience of cancer (having had it themselves or known a close friend or family member to have cancer) are significantly less willing to take these risks. However, the observable differences between individuals do not have much significance in general, 
and yet individuals in the survey sessions appeared to approach the questions quite differently.

The potential for unobserved heterogeneity between the individuals in the study prompts the use of fixed effects analysis. Models 3 and 4 provide fixed effects analysis with robust standard errors. Individual-specific differences account for about $24 \%$ of the variance in the data. In model (3), which replicates the OLS regression without the squared terms, the context dummy is significant at the $10 \%$ confidence level, and the coefficient is larger than in the pooled OLS analysis. However, the coefficients on the latency and morbidity differences retain their sign and significance, and their magnitudes are largely unaltered. Model (4) gives the fixed effects including the squared terms for time until death and morbidity differentials, finding these to be again opposite in sign to the main effect and significant at the $1 \%$ level of confidence. This again supports the finding of diminishing influence of time and of morbidity on the logged relativity.

Overall, the regression analysis corroborates the evidence from the central tendencies in supporting the proposed relationship between the VSL for cancer and the VSL for road accident fatality risks. While the context effect itself is not statistically significant at the 5\% level of confidence, the morbidity effect is significant, suggesting that morbidity drives much of the preference for the avoidance of cancer risks. We have shown that longer periods of latency reduce the VSL, ceteris paribus. Both of these effects are tempered through their diminishing marginal effects on the VSL.

Through central tendency and regression analysis we have contributed a range of new insights concerning the cancer premium, and provided evidence in support of some existing intuitions. Isolating latency, morbidity and context has allowed us to verify that latency and context effects offset one another in the comparison of road and cancer fatality risks. This provides support to our conjecture that the lack of agreement in the literature about the presence or absence of a cancer premium might relate to differences in the latency and morbidity specifications of the cancers used. We can, in addition, quantify the effects. The regressions show that, in our study, an additional year until death will reduce the relativity by between 14 and $15 \%$ (because the exponents of the coefficients are 0.850 and 0.857 . An additional month of morbidity increases the relativity by between 13 and $14 \%$. However, when we control for the diminishing effects of these variables by using squared terms, the absolute magnitude of the coefficients increase, offset by the opposite signed coefficients on the squared terms.

To summarise, we have shown that additional morbidity periods prior to fatality increase the relativity at a decreasing rate, and that latency offsets this morbidity effect by reducing the relativity again at a decreasing rate. As such, relativities for cancers with long morbidity periods and low latency periods are likely to be high, while relativities for cancers with short morbidity and long latency are likely to be low.

\subsection{Reconstructing the VSL from latency and morbidity parameters}

The information gleaned from the regression analyses can be recombined to provide a menu of relativities between cancer and road accident fatalities, given the latency and morbidity characteristics of the cancer fatality. For example, for a cancer fatality with 15 months' morbidity and 8 years' latency, the regression output from model (4) would suggest a relativity of 1.23 . For a sooner cancer (latency 5 years) with longer morbidity 
(24 months) the predicted relativity would rise to 3.21. Conversely, a cancer with 22 years' latency and just 9 months' morbidity would generate a predicted relativity of just 0.23 .

To capture these intuitions, we represent the relationships estimated in the Fixed Effects model (4) as a matrix in Table 7. In this representation, the numbers in the cells represent the relativity of cancer with given time until death and given morbidity periods, relative to an immediate, instantaneous road accident. The inputs to the calculation of this value are the time until death and morbidity difference from the instantaneous road accident fatality, measured in years and in months, respectively. The difference between the rows and the columns in the matrices are not uniform, reflecting the non-linear relationship between the relativity and the latency and morbidity periods. Nonetheless, the highest relativities correspond to the area with low latency and relatively high morbidity, and the lowest relativities are found where latency is long and morbidity periods are short.

To illustrate these intuitions further, we represent the relationships estimated in the Fixed Effects model (4) as a surface in Fig. 2. In this three-dimensional representation, the vertical axis represents the natural $\log$ of the relativity of cancer with given latency and morbidity periods, relative to an immediate, instantaneous road accident.

The axes along the horizontal plane represent the difference in the time until death, and morbidity difference from the instantaneous road accident fatality, measured in years and in months, respectively. The points highlighted on the surface represent the three cases outlined above. To find the relativity, one would simply take the exponent of the logged relativity elicited from reading the graph.

As discussed for the matrix, the slope of the graph is not uniform, which reflects the difference between the rate at which the latency and morbidity periods influence the relativity, and the rate at which these effects diminish.

\section{Discussion and Conclusions}

This paper investigated the relationship between the value of preventing a statistical cancer fatality and the value of preventing a statistical instantaneous road accident fatality. We proposed that the context of cancer would increase the relativity and the

Table 7 Matrix of reconstructed relativities comparing risks of cancer with specified morbidity and time until death, relative to instantaneous road accident fatality risks

\begin{tabular}{|c|c|c|c|c|c|c|}
\hline \multirow[b]{2}{*}{ Relativity } & & \multicolumn{5}{|c|}{ Morbidity (y) } \\
\hline & & 0 & 6 & 12 & 18 & 24 \\
\hline \multirow[t]{6}{*}{ Time until death (y) } & 0 & 0.744 & . & . & . & . \\
\hline & 5 & 0.12 & 0.647 & 0.196 & 3.35 & 3.212 \\
\hline & 10 & 0.035 & 0.19 & 0.577 & 0.984 & 0.944 \\
\hline & 15 & 0.019 & 0.102 & 0.309 & 0.527 & 0.505 \\
\hline & 20 & 0.018 & 0.099 & 0.301 & 0.514 & 0.493 \\
\hline & 25 & 0.033 & 0.176 & 0.535 & 0.938 & 0.875 \\
\hline
\end{tabular}




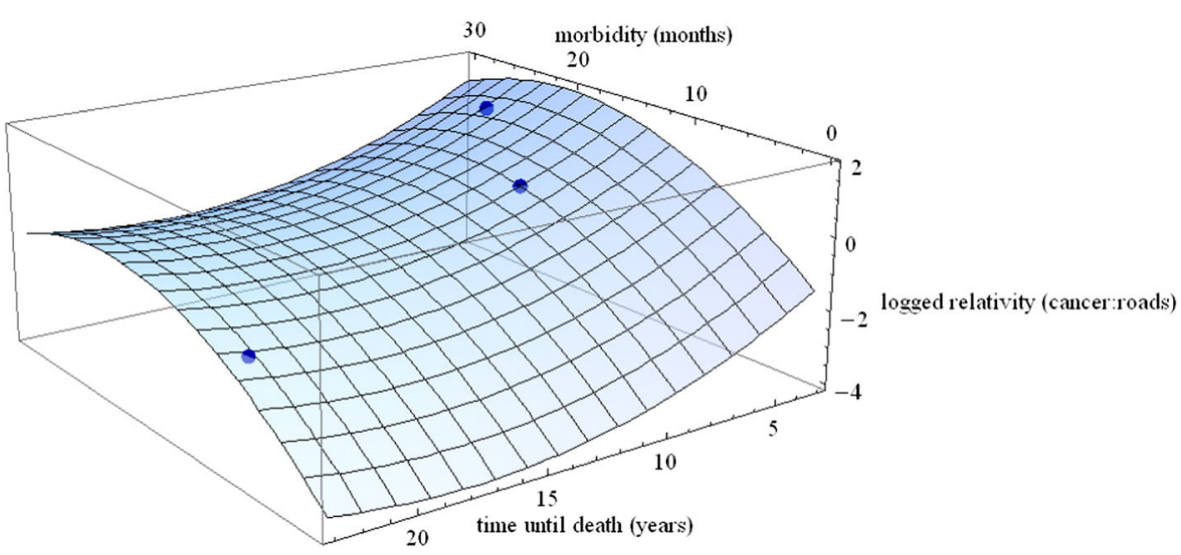

Fig. 2 Surface representing the influence of time until death and morbidity on the logged relativity between the VSL for cancer and for an immediate, instantaneous road accident fatality

latency associated with cancer would decrease it. This was verified using a Risk-Risk trade-off study designed to isolate the impact of context, morbidity and latency in determining the overall cancer premium. The central tendencies indicated a good degree of consistency over the attributes of the fatality scenarios that we investigated. Regression analysis supported the proposed relationship, suggesting that morbidity increases the VSL, and latency decreases it, with morbidity driving the context premium in these data. This analysis allowed us to estimate the size of the morbidity and time until death effects for our sample. Both effects are non-linear, with diminishing marginal effects on the VSL. It also allowed the introduction of demographic heterogeneity in the affected populations of different cancers, and fixed effects were used to control for unobserved individual differences.

The usefulness of these results was demonstrated in a policy-relevant application, where information about latency (via the time until death) and morbidity associated with a particular type of cancer fatality can be used to reconstruct its appropriate VSL (relative to an instantaneous road accident fatality for which the value is well established). As such, the applicability of a "cancer premium" can be addressed on a case-by-case basis. Typically, where the latency period is short and the morbidity period is long, a case can be made for increasing the VSL and allocating more resources to the prevention of the cancer than when the cancer fatality would be preceded only by a short illness and would occur many years from now. The practicalities of such an approach are admittedly not straightforward, and as such we provide a simpler policy interpretation of our results in Appendix 2, where we provide an approach that combines just two $\mathrm{C}_{\mathrm{T}} \mathrm{R}_{\mathrm{t}}$ relativities and generates estimates of the effective discount rate $r$ and the context premium $(1+x)$, as we outlined in Eq. (1). We can then test the validity of the application of a " $\times 2$ " cancer premium for cancer fatalities. Through these two methods, the framework is now in place for respecting individuals' preferences when making policy decisions that influence the risk of fatality from cancers with different latency and morbidity periods.

In summary, we have proposed, verified and demonstrated a structural relationship between cancer and road accident risk valuations. We have conducted a rich analysis which can encompass a broad variety of cancer morbidity and latency periods and so 
provide a fairly comprehensive framework for considering the likely relative value of cancers with different characteristics. This approach provides a framework within which to extend future discussion of cancer valuations, which is flexible and capable of accommodating the introduction of different discounting hypotheses, cancer characteristics and latency periods and which could help to reconcile the varied VSL estimates for cancer that characterise the current literature. We also hope that the application of cancer valuations can be less arbitrary and better tailored to the specific properties of the cancer scenario under valuation through the insights generated by this research.

\section{Appendix 1}

\section{Survey text extract describing cancer}

Symptoms of cancer were derived from the American Cancer Association website and chosen to be as specific as possible without losing generality by becoming cancer-specific.

"We are interested in those cancers that are caused by exposure to harmful substances that you come across on a day-to-day basis, for example at work or from near to where you live. They are NOT caused by lifestyle choices like smoking or drinking to excess, or solely by genetics. Please notice that this distinction means that your personal risk level is unlikely to differ much from the average risk.

"Another important point is that we are talking about cancers where the chance of survival is extremely small and we shall treat them as terminal. Please be aware that although cures for some types of cancer might be developed over time, it is extremely unlikely that a cure would be found for all of the cancers we are thinking about.

"Also, there are a wide variety of cancers and they all have different characteristics. We can't ask about every cancer separately, so instead we'll try to cover as many as we can using groups of cancers with similar characteristics.

"For the cancers we are concerned with, the symptoms might include unexplained weight loss, having fevers and feeling generally unwell, and also having less energy than before. You will have some pain and might need to be treated using drugs that make you sick.

"You would go through stages of illness, each one a bit more severe than the one before it. It is hard to be precise about how bad the symptoms would be, but usually they get worse as time passes. A longer time with symptoms means you would be in each stage of the illness for a bit longer.

"These are the symptoms of a typical cancer case, and you should imagine that this is what it would be like for you." 


\section{Appendix 2}

\section{A simple policy application}

The analysis in the main text used all questions and all information to generate a detailed account of the effects of latency and morbidity on the VSL for cancer. This clearly offers a new and rich set of information for allocative policy decisions. However, in reality it is not practically feasible for policymakers to use a different valuation for each cancer case. Given this limitation, we estimate some key underlying parameters for policy use that will simplify the approach to understanding the VSL $_{\text {CANCER }}$. This also allows us to test the validity of the application of a multiplier of 2, which doubles the VSL for cancer compared to a road accident VSL in UK government policy. We can use the $C_{T} R_{t}$ relationship proposed in Eq. (1) and verified through the regression analysis to simplify the information from just two relativities into a context premium (including a fixed morbidity period of 12 months) and an effective discount rate capturing the effect of latency. In addition to the benefit of simplifying the analysis of survey relativities, this procedure allows the inference of the effective discount rate simultaneously with the context premium, which is more informative than imposing an externally chosen discount rate.

While the previous analysis suggests that morbidity was important, and that the effect of latency might be non-linear, we prefer to keep the $\mathrm{C}_{\mathrm{T}} \mathrm{R}_{\mathrm{t}}$ relationship simple by incorporating morbidity into the overall context parameter and by accommodating the effect of latency through a conventional discounting procedure, as in Eq. (1). This allows us to elicit two key policy-relevant parameters, which are straightforward to explain and interpret and practically applicable in a policy setting. It also reduces the requirement for information in the survey scenarios used to elicit the parameters. It is possible to adapt the relationship in Eq. (1) to incorporate morbidity duration, but this can only be used in cases where the duration of illness is known.

From Eq. (1) it follows that:

$$
\frac{\mathrm{C}_{10}}{\mathrm{R}_{2}}=\frac{(1+\mathrm{x})}{(1+\mathrm{r})^{8}}
$$

and

$$
\frac{C_{25}}{R_{2}}=\frac{(1+x)}{(1+r)^{23}}
$$

so that from Eqs. (7) and (8):

$$
\frac{\mathrm{C}_{10} / \mathrm{R}_{2}}{\mathrm{C}_{25} / \mathrm{R}_{2}}=(1+\mathrm{r})^{15}
$$

Based on the trimmed geometric means of the $C_{10} / R_{2}$ and $C_{25} / R_{2}$ ratios from Q2 and Q3, it then follows from Eq. (9) that:

$$
r=0.0737
$$


and hence, by substituting for $r$ in Eqs. (7) or (8), that:

$$
\mathrm{x}=0.434
$$

The effective discount rate is therefore $7.37 \%$ per year and the context premium incorporating 12 months morbidity is 1.43 , implying that cancer fatality risk increases are perceived to be 1.43 times as bad as road accident risk increases which occur at the same time, given this illness period. These estimates are within a reasonable range given previous literature.

In this study, using the generic descriptions of cancer with 12 month morbidity, we found a context premium of 1.43 (i.e. the VSL for cancer is 1.43 times as high as the VSL for road accidents, when the fatalities would occur at the same time) and a discount rate of $7.37 \%$. In combination, these parameters mean that a latency period of ten years or more in the cancer case reduces the relative value of the latent cancer fatality and the current period road accident fatality to $1: 1$. Therefore, we find no evidence to support the application of a " $\times 2$ " cancer premium based on the preferences of this particular sample of the general public.

Open Access This article is distributed under the terms of the Creative Commons Attribution 4.0 International License (http://creativecommons.org/licenses/by/4.0/), which permits unrestricted use, distribution, and reproduction in any medium, provided you give appropriate credit to the original author(s) and the source, provide a link to the Creative Commons license, and indicate if changes were made.

\section{References}

Adamowicz, W., Dupont, D., Krupnik, A., \& Zhang, J. (2011). Valuation of cancer and microbial disease risk reductions in municipal drinking water: An analysis of risk context using multiple valuation methods. Journal of Environmental Economics and Management, 61, 213-226.

Alberini, A., \& Scasny, M. (2010). Does the cause of death matter? The effect of dread, controllability, exposure and latency on the VSL. FEEM working paper No. 139.2010.

Alberini, A., \& Scasny, M. (2011). Context and the VSL: Evidence from a stated preference study in Italy and the Czech Republic. Environmental \& Resource Economics, 49, 511-538.

Beattie, J., Covey, J., Dolan, P., Hopkins, L., Jones-Lee, M., Loomes, G., Pidgeon, N., Robinson, A., \& Spencer, A. (1998). On the contingent valuation of safety and the safety of contingent valuation: Part ICaveat investigator. Journal of Risk and Uncertainty, 17, 5-25.

Cameron, T. A., \& DeShazo, J. (2013). Demand for health risk reductions. Journal of Environmental Economics and Management, 65, 87-109.

Cameron, T. A., DeShazo, J. R., \& Johnson, E. H. (2009). Willingness to pay for health risk reductions: Differences by type of illness. University of Oregon working paper.

Chilton, S., Jones-Lee, M., Kiraly, F., Metcalf, H., \& Pang, W. (2006). Dread risks. Journal of Risk and Uncertainty, 33, 165-182.

Covey, J. A. (2001). People's preferences for safety control: Why does baseline risk matter? Risk Analysis, 21, $331-340$.

Davis, L. W. (2004). The effect of health risk on housing values: Evidence from a cancer cluster. American Economic Review, 94, 1693-1704.

European Commission. (2001). Recommended Interim Values for the Value of Preventing a Fatality in DG Cost Benefit Analysis [Online]. Available at: http://ec.europa.eu/environment/enveco/others/pdf/recommended interim_values.pdf (Accessed 20 May 2014).

Frederick, S., Loewenstein, G., \& O’Donoghue, T. (2002). Time discounting and time preference: A critical review. Journal of Economic Literature, 40(2), 351-401. 
Gayer, T., Hamilton, J. T., \& Viscusi, W. K. (2000). Private values of risk tradeoffs at Superfund sites: Housing market evidence on learning about risk. Review of Economics and Statistics, 82, 439-451.

Gayer, T., Hamilton, J. T., \& Viscusi, W. K. (2002). The market value of reducing cancer risk: Hedonic housing prices with changing information. Southern Economic Journal, 69, 266-289.

Hammitt, J. K., \& Haninger, K. (2010). Valuing fatal risks to children and adults: Effects of disease, latency, and risk aversion. Journal of Risk and Uncertainty, 40, 57-83.

Hammitt, J. K., \& Liu, J. T. (2004). Effects of disease type and latency on the value of mortality risk. Journal of Risk and Uncertainty, 28, 73-95.

Health and Safety Executive (2001). Reducing risks, protecting people. HSE Books.

Jones-Lee, M. W. (1989). The economics of safety and physical risk. Oxford: Basil Blackwell.

Jones-Lee, M. W., Hammerton, M., \& Philips, P. R. (1985). The value of safety: Results of a national sample survey. Economic Journal, 95, 49-72.

Magat, W. A., Viscusi, W. K., \& Huber, J. (1996). A reference lottery metric for valuing health. Management Science, 42, 1118-1130.

McDaniels, T. L., Kamlet, M. S., \& Fischer, G. W. (1992). Risk perception and the value of safety. Risk Analysis, 12, 495-503.

Organisation for Economic Co-operation and Development (2012). Mortality risk valuation in environment, health and transport policies. OECD Publishing.

Pratt, J. W., \& Zeckhauser, R. (1996). Willingness to pay and the distribution of risk and wealth. The Journal of Political Economy, 104, 747-763.

Rushton, L., Bagga, S., Bevan, R., Brown, T., Cherrie, J., Holmes, P., Hutchings, S., Fortunato, L., Slack, R., \& Van Tongeren, M. (2010). The burden of occupational cancer in Great Britain. Research Report RR800. Health and Safety Executive. 2010. Available at: http://www.hse.gov.uk/research/rrpdf/rr800.pdf (Accessed 19 May 2010).

Samuelson, P. A. (1937). A note on measurement of utility. The Review of Economic Studies, 4, 155-161.

Slovic, P. (1987). Perception of risk. Science, 236, 280-285.

Tsuge, T., Kishimoto, A., \& Takeuchi, K. (2005). A choice experiment approach to the valuation of mortality. Journal of Risk and Uncertainty, 31, 73-95.

Van Houtven, G., Sullivan, M. B., \& Dockins, C. (2008). Cancer premiums and latency effects: A risk tradeoff approach for valuing reductions in fatal cancer risks. Journal of Risk and Uncertainty, 36, 179-199.

Viscusi, W. K. (1998). Rational risk policy. Oxford, Oxford university press.

Viscusi, W. K. (2013). The value of individual and societal risks to life and health. In M. Machina \& W. K. Viscusi (eds.), Handbook of the Economics of Risk and Uncertainty. Newnes.

Viscusi, W. K., Magat, W. A., \& Huber, J. (1991). Pricing environmental-health risks: Survey assessments of risk-risk and risk-dollar trade-offs for chronic bronchitis. Journal of Environmental Economics and Management, 21, 32-51.

Viscusi, W. K., Huber, J., \& Bell, J. (2014). Assessing whether there is a cancer premium for the value of a statistical life. Health Economics, 23, 384-396. 